\title{
Integrated Use of Potassium and Soil Mulching on Growth and Productivity of Garlic (Allium sativum L.) under Deficit Irrigation
}

\author{
Mohammed A. Barakat ${ }^{1, a}$, Ashraf S. Osman ${ }^{1, b}$, Wael M. Semida ${ }^{1, c^{*}}$ and \\ Mohammed A. Gyushi, ${ }^{1, d}$ \\ ${ }^{1}$ Hort. Department, Faculty of Agriculture, Fayoum University, Fayoum, Egypt \\ amas11@fayoum.edu.eg, bashraf@fayoum.edu.eg, 'cwms00@fayoum.edu.eg, \\ dmah09@fayoum.edu.eg \\ ${ }^{*}$ Corresponding author
}

Keywords: garlic (Allium sativum L.), deficit irrigation, potassium, soil mulching.

\begin{abstract}
This study was carried out during the winter seasons of 2016 and 2017 at private farm in Beni Suief Governorate, Egypt to investigate the integrative use of potassium levels (24, 48, 72 and $96 \mathrm{~kg} \mathrm{~K}_{2} \mathrm{O}$ fed $^{-1}$ ) and soil mulching (rice straw and bare soil) on growth and productivity of garlic under three levels of surface irrigation $\left(60,80\right.$ and $100 \%$ of crop evapotranspiration $\left(\mathrm{ET}_{\mathrm{C}}\right)$ ). The experimental layout was a spit-spilt plot system based on Randomized Complete Blocks Design. Irrigation levels, potassium rates and mulch types were randomly allocated in the main, sub and subsub plots, orderly. The obtained results showed that, the amount of irrigation at $80 \mathrm{and} / \mathrm{or} 100 \% \mathrm{ET}_{\mathrm{C}}$ was accompanied increases by plant height, leaves number, leaf area and dry weight plant ${ }^{-1}$, total bulbs yield, individual bulb weight, cloves weight and number bulb ${ }^{-1}$ compared to irrigation $60 \%$ $E_{C}$. The application of potassium from 24 to 48 and further to 72 and $96 \mathrm{~kg} \mathrm{~K}_{2} \mathrm{O}$ fed $^{-1}$ gave gradual increments in the aforementioned growth and productivity of traits. The rice straw mulch augmented all previous growth, total bulbs yield and its components compared to bare soil. Generally, the $1^{\text {st }}$ order interaction between irrigation percent at 100 and/or $80 \% \mathrm{ET}_{\mathrm{C}} \times$ potassium rate at 96 and/or $72 \mathrm{~kg} \mathrm{~K}_{2} \mathrm{O}$ fed $^{-1}$, irrigation percent at 80 and/or $100 \% \mathrm{ET}_{\mathrm{C}} \times$ rice straw, potassium rate at $96 \mathrm{~kg} \mathrm{~K}_{2} \mathrm{O}$ $\mathrm{fed}^{-1} \times$ rice straw and the $2^{\text {nd }}$ interaction of irrigation percent at $100 \% \mathrm{ET}_{\mathrm{C}} \times$ potassium rate at $96 \mathrm{~kg}$ $\mathrm{K}_{2} \mathrm{O}$ fed $^{-1} \times$ rice straw mulch had pronounced effects on morphological characters and bulbs yield and its components compared to other combined treatments.
\end{abstract}

\section{Introduction}

Garlic (Allium sativum L.) is one of main vegetable crops and it has been used for flavoring, soup, sausages and salads, in addition to its medical value [1]. Increasing morphological traits and productivity of garlic are essential aims for both growers and consumers. These aims depend on many environmental factors such as irrigation, potassium, mulch and other agricultural practices. The relative amount of water available to agriculture is declining worldwide due to the rapid population growth and the greater incidence of drought in recent years caused by climate change and different human activities [2]. The challenge of irrigated agriculture in our time is how to produce more morphological and productivity of garlic crops from limited water supply. Working on surface irrigation on garlic, [3] they reported that, 75 and $100 \%$ ETp (potential evapotranspiration determined by class A pan) was at par in plant height and cured bulb diameter, in $2^{\text {nd }}$ season, the reverse was true in total bulbs yield, fresh and cured bulb weight. Also, increasing furrow irrigation from $40 \% \mathrm{ET}_{\mathrm{C}}$ to $100 \% \mathrm{ET}_{\mathrm{C}}$, significantly, increased onion bulbs yield and bulb diameter whereas, irrigation; 40 , $55,70,80$ and $100 \% \mathrm{ET}_{\mathrm{C}}$ did reflect any appreciable effect on bulb weight [4] .

Potassium is assumed to fulfill many tasks in some physiological and biochemical processes for the growth and productivity of plant. [5] displayed that, increasing potassium rate from 0 to 24 and further to 48,72 and $96 \mathrm{~kg} \mathrm{fed}^{-1}$ to grown garlic plants correspondingly and intrinsically increased plant height, leaves number and area plant ${ }^{-1}$, plant dry weight (without roots) and blubbing ratio. [6] reported that, applied potassium rate at 100 and/or $125 \mathrm{~K}_{2} \mathrm{O} \mathrm{fed}{ }^{-1}$, significantly, had maximum leaves number and total dry weight plant $^{-1}$ on garlic however, all potassium levels $(0,25,50,75,100$ and 
$125 \mathrm{~kg} \mathrm{~K}_{2} \mathrm{O}$ fed $^{-1}$ ) did not reflect any, significant, effect on plant height. [7] displayed that, there was a close relationship between applied potassium rates and garlic productivity. In this regard, [8] reteraved that, application $300 \mathrm{~K}_{2} \mathrm{O}$ fed $^{-1}$ to grown of garlic plants was a pioneer in total bulbs yield, cloves number bulb ${ }^{-1}$ and clove weight relative to the lower levels (control and $100 \mathrm{~K}_{2} \mathrm{O} \mathrm{fed}^{-1}$ ).

Numerous investigators have documented the benefit of rice straw mulch on morphological and productivity. Concerning the effect of rice straw mulch on the morphological phenomena, [9] illustrated that, plant height, leaves number plant ${ }^{-1}$, pseudo stem diameter and root dry matter on onion were, significantly, higher with rice straw compared to bare soil. Regarding the influence of rice straw mulch on the productivity of garlic, [10] showed that, higher total bulbs yield, fresh weight bulb $^{-1}$, cloves number bulb ${ }^{-1}$ and total bulbs yield plot $^{-1}$ than bare soil.

Therefore, this study was investigated to the influence of furrow irrigation percent, potassium level and mulch type on morphological traits and productivity phenomena.

\section{Materials and Methods}

Two similar field experiments at a private farm, Beni Suef Governorate, Egypt during the winter seasons of 2016 and 2017 to recognize the effect of three surface irrigation percent's; 60, 80 and $100 \% \mathrm{ET}_{\mathrm{C}}$, four potassium rates; 24, 48, 72 and $96 \mathrm{~kg} \mathrm{~K}_{2} \mathrm{O}$ fed ${ }^{-1}$ and two mulch types; rich straw mulch and bare soil on garlic crop cv. Sids 40. The experimental layout was a split-split plot based on a Randomized Complete Blocks Design with three replications. Irrigation percent's, potassium rates and mulch types were randomly allocated in the main, sub- and sub-sub plots, orderly. Each experimental unit including 3 rows; $3.5 \mathrm{~m}$ long and $70 \mathrm{~cm}$ wide. Each two adjacent main plots were isolated by $2 \mathrm{~m}$ and two adjacent sub-plots were separated with $0.5 \mathrm{~m}$. Before the initiation of experimental site, soil samples were collected and analyses according to the published standard [11]. Results of soil analyses have showed, the soil texture; clay, $\mathrm{PH} ; 7.41$ and $\left.7.63, \mathrm{~N} \mathrm{(mg} \mathrm{kg}{ }^{-1}\right) ; 9$ and 9.33, $\mathrm{P}\left(\mathrm{mg} \mathrm{kg}^{-1}\right)$; 9.52 and 9.70 and $\mathrm{K}\left(\mathrm{meg} \mathrm{kg}^{-1}\right)$; 289 and 301 in 2016 and 2017, respectively. Nearly cloves of the same size were soaked in running tap water for 12 hours prior to sowing, then were hand planted singly in both sides of inter-row spacing $10 \mathrm{~cm}$ on September $24^{\text {th }}$ and $30^{\text {th }}$ in 2016 and 2017, respectively.

\section{Irrigation percent treatments}

The growing garlic plants were started different irrigation treatments after 30 days from planting and after that were irrigated every 15 days interval. Three irrigation water levels; 60,80 and $100 \% \mathrm{ET}_{\mathrm{C}}$ were applied where $\mathrm{ET}_{\mathrm{C}}$ represents the crop water evapotranspiration. The crop evapotranspiration $\left(\mathrm{ET}_{\mathrm{C}}\right)$ were estimated using the crop coefficient according to the following equation

$$
\mathrm{ETC}=\mathrm{ETO} \times \mathrm{Kc}
$$

Where:

$\mathrm{ET}_{\mathrm{C}}=$ crop water evapotranspiration $\left(\mathrm{mm} \mathrm{d}^{-1}\right)$.

$\mathrm{ET}_{\mathrm{O}}=$ reference evapotranspiration of crop; average monthly of Beni Suef, Egypt for 3.25, 2.59, 2.11, 1.96, 2.51 and 3.36 for October, November, December, January, February and March.

$\mathrm{K}_{\mathrm{C}}=$ crop coefficient.

The amount of irrigation water applied to each plot during the irrigation treatment was determined by using the following equation

$$
\mathrm{IWA}=\frac{\mathrm{A} \times \mathrm{ETC} \times \mathrm{Ii}}{\mathrm{Ea} \times 1000}+\mathrm{LR}
$$

Where:

IWA $=$ irrigation water applied $\left(\mathrm{m}^{3}\right)$.

$\mathrm{ET}_{\mathrm{C}}=$ crop evapotranspiration $\left(\mathrm{mm} \mathrm{d}^{-1}\right)$.

$A=$ plot area $\left(\mathrm{m}^{2}\right)$.

$\mathrm{Ea}=$ application efficiency $(\%)(\mathrm{Ea}=60 \%)$.

$\mathrm{Ii}=$ irrigation intervals $(\mathrm{d})$.

$\mathrm{LR}=$ leaching requirements $\left(\mathrm{m}^{3}\right)$ 
The amount of irrigation water applied (IWA) was controlled through plastic pipe of $50 \mathrm{~mm}$ diameter. The amount of water delivered through a plastic pipe was calculated using the following equation according to [12].

$$
Q=C A \sqrt{2 g h} \times 10^{-3}
$$

Where:

$\begin{array}{lll}\mathrm{Q} & =\text { discharge of irrigation water }\left(1 . \mathrm{sec}^{-1}\right), & \mathrm{C}=\text { coefficient of discharge } \\ \mathrm{A} & =\text { cross section area of irrigation pipe }\left(\mathrm{cm}^{2}\right) & \mathrm{G}=\text { gravity acceleration }\left(\mathrm{cm} . \mathrm{sec}^{-2}\right) \\ \mathrm{H} & =\text { average effective head of water }(\mathrm{cm}) . & \end{array}$

\section{Potassium rate treatments}

The form of potassium fertilizer level was potassium sulfate fertilizer $\left(48 \% \mathrm{~K}_{2} \mathrm{O}\right)$ and was side banded in three equal portions; 60, 90 and 120 days after seed sowing.

\section{Mulch type treatments}

Rice straw mulch was done immediately after complete earthing (about 7 days from seed sowing) and bare soil uncovered with rice straw.

All experimental unites received identical doses of $\mathrm{N}$ and $\mathrm{P}_{2} \mathrm{O}_{5}$ at 100 and $60 \mathrm{Kg}$ fed $^{-1}$, orderly and all other agro-management practices were achieved as recommended in the commercial production of garlic. Harvesting was done after 180 days from planting, in both seasons. In each experimental unit, plants of the middle row were allocated for morphological measurements while, plants of the two outer rows were allocated to measure bulbs yield and its components.

\section{Data Recorded}

Morphological characters was done 160 days after planting

1. Plant height (cm); measured from the bulb bottom to the tip of longest leaf.

2. Leaves number plant $^{-1}$; estimated by counts.

3. Leaves area plant ${ }^{-1}\left(\mathbf{c m}^{2}\right)$; calculated using relationship between fresh weight of leaves plant ${ }^{-}$ ${ }^{1}$ and fresh weight and area of 20 disks taken from fresh leaves by a borer known its diameter [13].

4. Dry weight plant ${ }^{-1}$ without roots (g); plants sample were placed in a forced-oven at $70 \mathrm{C}^{\mathrm{o}}$ till the weights became constants.

Blubs yield and its components were achieved 180 days after planting

1. Total bulbs yield fed ${ }^{-1}$ (tones); bulbs in each treatment were weighed and converted to total bulbs yield fed $^{-1}$.

2. Bulb weight (g); calculated by the following equation

Bulb weight $=\quad$ bulbs weight in each experimental unit

bulbs number in each experimental unit

3. Cloves weight $\mathbf{b u l b}^{-1}(\mathrm{~g})$; ten bulbs from each treatment were separated individually into cloves bulb $^{-1}$ and weighed and the average cloves weight bulb ${ }^{-1}$ was calculated.

4. Cloves number bulb ${ }^{-1}$; using ten bulbs, from each treatment, were separated individually into cloves, counted and average cloves number bulb ${ }^{-1}$ was calculated.

5. Average clove weight (g); calculated by the following formula

$$
\text { Average clove weight }=\quad \frac{\text { weight cloves bulb-1 }}{\text { number of cloves bulb }}
$$

\section{Statistical analysis}

Appropriate analysis of variance on obtained data was performed. Comparisons among mean treatments were carried out using Revised Least Significant Difference at $\mathrm{P} \leq 0.05$ [14]. 


\section{Results}

\section{Morphological Characters}

Table 1 shows the main effects of three studied factors on morphological phenomena, during the winter seasons of 2016 and 2017. Irrigation at 80 and/or $100 \% \mathrm{ET}_{\mathrm{C}}$ produced higher, significantly, than irrigation at $60 \% \mathrm{ET}_{\mathrm{C}}$ on plant height, leaves number and leaves area plant ${ }^{-1}$ while, increasing irrigation from 60 to 80 and further to $100 \%$ ETC, truly, increased whole plant dry weight, in both seasons. Application potassium 24 up to $96 \mathrm{~kg} \mathrm{~K}_{2} \mathrm{O}$ fed $^{-1}$ accompanied, significantly, increased, on plant height, leaves number, leaves area and dry weight plant ${ }^{-1}$, in both seasons whilst, difference potassium between 72 and $96 \mathrm{~kg} \mathrm{~K}_{2} \mathrm{O}$ fed ${ }^{-1}$ in plant height and 48 and $72 \mathrm{~kg} \mathrm{~K}_{2} \mathrm{O}$ fed ${ }^{-1}$ in leaves number plant ${ }^{-1}$, in $1^{\text {st }}$ season, was at par. Significant increments of rice straw mulch over bare soil on plant height, leaves number, leaves area and whole dry weight plant $^{-1}$, in both investigated seasons.

The statistical comparisons listed in Table 2 show that, the effect of $1^{\text {st }}$ order interaction between irrigation percent's and potassium rates on vegetative traits was, significant, in both seasons nevertheless, leaves number plant ${ }^{-1}$ did not appear to be significantly, in $2^{\text {nd }}$ season. The application of irrigation at $80 \mathrm{and} / 100 \% \mathrm{ET}_{\mathrm{C}}$ together with potassium at 72 and $/$ or $96 \mathrm{~kg} \mathrm{K2O} \mathrm{fed}{ }^{-1}$ had maximum values of plant height, leaves number, area and dry weight plant ${ }^{-1}$. Also, the results obtained of interaction between irrigation 80 and/or $100 \% \mathrm{ET}_{\mathrm{C}} \times$ rice straw mulch had, truly, the maximum leaves area plant-1, in both seasons while, the interaction between $100 \% \mathrm{ET}_{\mathrm{C}} \times$ rice straw mulch was the best on plant height and dry weight plant $^{-1}$. The application of all potassium rates $\times$ all mulch types did not reflect any appreciable effect on studied growth characters except dry weight plant ${ }^{-1}$, in both seasons. The results indicated that, in both seasons within $96 \mathrm{~kg} \mathrm{~K}_{2} \mathrm{O} \mathrm{fed}^{-1}$, the application of rice straw mulch resulted in heaviest dry weight plant ${ }^{-1}$.

Table 1. The main effect of irrigation percent, potassium rate and mulch type on plant height $(\mathrm{cm})$, leaves number, leaves area $\left(\mathrm{cm}^{2}\right)$, leaves dry weight $(\mathrm{g})$ and dry weight plant ${ }^{-1}(\mathrm{~g})$ during the winter seasons of 2016/2017 and 2017/2018

\begin{tabular}{|c|c|c|c|c|c|c|c|c|}
\hline \multirow{2}{*}{$\begin{array}{l}\text { Treatments } \\
\text { Irrigation } \\
(\% \text { ETc) }\end{array}$} & \multicolumn{4}{|c|}{2016} & \multicolumn{4}{|c|}{2017} \\
\hline & $\begin{array}{c}\text { Plant } \\
\text { Height }\end{array}$ & $\begin{array}{c}\text { Leaves } \\
\text { Number } \\
\text { Plant }^{-1}\end{array}$ & $\begin{array}{c}\text { Leaves } \\
\text { area } \\
\text { Plant }^{-1}\end{array}$ & $\begin{array}{c}\text { D.W. } \\
\text { Plant }^{-1}\end{array}$ & $\begin{array}{l}\text { Plant } \\
\text { height }\end{array}$ & $\begin{array}{c}\text { Leaves } \\
\text { Number } \\
\text { Plant }^{-1}\end{array}$ & $\begin{array}{c}\text { Leaves } \\
\text { area } \\
\text { Plant }^{-1}\end{array}$ & $\begin{array}{l}\text { D.W. } \\
\text { Plant }^{-1}\end{array}$ \\
\hline 100 & $95.12^{\mathrm{A}^{\bullet}}$ & $9.40^{\mathrm{A}}$ & $558.14^{\mathrm{B}}$ & $45.53^{\mathrm{A}}$ & $97.57^{\mathrm{A}}$ & $9.41^{\mathrm{A}}$ & $61.42^{\mathrm{A}}$ & $48.51^{\mathrm{A}}$ \\
\hline 80 & $95.03^{\mathrm{A}}$ & $9.43^{\mathrm{A}}$ & $595.05^{\mathrm{A}}$ & $41.45^{\mathrm{B}}$ & $97.66^{\mathrm{A}}$ & $9.39^{\mathrm{A}}$ & $58.79^{\mathrm{B}}$ & $42.12^{\mathrm{B}}$ \\
\hline 60 & $80.23^{\mathrm{B}}$ & $8.23^{\mathrm{B}}$ & $347.62^{C}$ & $23.09^{C}$ & $84.61^{\mathrm{B}}$ & $8.30^{\mathrm{B}}$ & $42.97^{\mathrm{C}}$ & $25.90^{\mathrm{C}}$ \\
\hline \multicolumn{9}{|l|}{$\begin{array}{l}\text { Potassium } \\
\left(\mathrm{K}_{2} \mathrm{O} \text { fed }^{-1}\right)\end{array}$} \\
\hline 24 & $81.81^{\mathrm{C}}$ & $8.40^{\mathrm{C}}$ & $363.28^{\mathrm{D}}$ & $26.98^{\mathrm{D}}$ & $40.83^{\mathrm{D}}$ & $8.37^{\mathrm{D}}$ & $388.12^{\mathrm{D}}$ & $25.54^{\mathrm{D}}$ \\
\hline 48 & $90.91^{\mathrm{B}}$ & $8.97^{\mathrm{B}}$ & $494.31^{\mathrm{C}}$ & $35.85^{\mathrm{C}}$ & $44.84^{C}$ & $8.89^{\mathrm{C}}$ & $470.41^{\mathrm{C}}$ & $35.34^{\mathrm{C}}$ \\
\hline 72 & $93.80^{\mathrm{A}}$ & $9.14^{\mathrm{B}}$ & $560.47^{\mathrm{B}}$ & $38.67^{\mathrm{B}}$ & $46.68^{\mathrm{B}}$ & $9.27^{\mathrm{B}}$ & $535.27^{\mathrm{B}}$ & $43.07^{\mathrm{B}}$ \\
\hline 96 & $93.97^{\mathrm{A}}$ & $9.57^{\mathrm{A}}$ & $583.02^{\mathrm{A}}$ & $45.26 \mathrm{~A}$ & $48.63^{\mathrm{A}}$ & $9.61^{\mathrm{A}}$ & $598.82^{\mathrm{A}}$ & $51.42^{\mathrm{A}}$ \\
\hline \multicolumn{9}{|l|}{ Mulch (type) } \\
\hline Bare soil & $89.11^{\mathrm{B}}$ & $8.84^{\mathrm{B}}$ & $469.89^{\mathrm{B}}$ & $32.61^{\mathrm{B}}$ & $91.72^{\mathrm{B}}$ & $8.90^{\mathrm{B}}$ & $477.64^{\mathrm{B}}$ & $36.05^{\mathrm{B}}$ \\
\hline Rice straw & $91.13^{\mathrm{A}}$ & $9.20^{\mathrm{A}}$ & $530.65^{\mathrm{A}}$ & $40.77^{\mathrm{A}}$ & $94.83^{\mathrm{A}}$ & $9.17^{\mathrm{A}}$ & $518.67^{\mathrm{A}}$ & $41.64^{\mathrm{A}}$ \\
\hline
\end{tabular}

-Values marked with the same letter(s) within the main effect are statistically similar using Revised LSD. Test at probability $=0.05$. Uppercase refer to the main effect. 
Table 2. The $1^{\text {st }}$ interaction of irrigation percent, potassium rate and mulch type on plant height $(\mathrm{cm})$, leaves number, leaves area $\left(\mathrm{cm}^{2}\right)$ and dry weight plant ${ }^{-1}(\mathrm{~g})$ during the winter seasons of 2016/2017 and 2017/2018

\begin{tabular}{|c|c|c|c|c|c|c|c|c|c|c|}
\hline \multicolumn{2}{|c|}{ Treatments } & \multicolumn{3}{|c|}{2016} & \multicolumn{5}{|c|}{2017} & \multirow[b]{2}{*}{$\begin{array}{l}\text { dry } \\
\text { weight } \\
\text { plant }^{-1}\end{array}$} \\
\hline I & $\mathbf{K}$ & M & $\begin{array}{l}\text { Plant } \\
\text { height }\end{array}$ & $\begin{array}{l}\text { leaves } \\
\text { number } \\
\text { plant }^{-1}\end{array}$ & $\begin{array}{l}\text { leaves } \\
\text { area } \\
\text { plant }^{-1}\end{array}$ & $\begin{array}{l}\text { dry } \\
\text { weight } \\
\text { plant }^{-1}\end{array}$ & $\begin{array}{l}\text { Plant } \\
\text { height }\end{array}$ & $\begin{array}{l}\text { leaves } \\
\text { number } \\
\text { plant }^{-1}\end{array}$ & $\begin{array}{l}\text { leaves } \\
\text { area } \\
\text { plant }^{-1}\end{array}$ & \\
\hline \multirow[t]{4}{*}{100} & 24 & & $84.90^{\mathrm{e}}$ & $8.67^{\mathrm{ef}}$ & $416.5^{g}$ & $29.33^{\mathrm{e}}$ & $84.37^{\mathrm{h}}$ & $8.83^{\mathrm{a}}$ & $482.1^{\mathrm{f}}$ & $33.92^{\mathrm{g}}$ \\
\hline & 48 & & $94.87^{\mathrm{c}}$ & $9.40^{\mathrm{bc}}$ & $565.4^{\mathrm{d}}$ & $49.78^{\mathrm{ab}}$ & $95.10^{\mathrm{de}}$ & $9.17^{\mathrm{a}}$ & $553.5^{\mathrm{d}}$ & $45.79^{e}$ \\
\hline & 72 & & $101.6^{\mathrm{a}}$ & $9.67^{\mathrm{ab}}$ & $637.3^{\mathrm{b}}$ & $51.33^{\mathrm{ab}}$ & $104.07^{\mathrm{b}}$ & $9.70^{\mathrm{a}}$ & $632.5^{\mathrm{b}}$ & $53.32^{\mathrm{c}}$ \\
\hline & 96 & & $99.10^{\mathrm{b}}$ & $9.87^{\mathrm{a}}$ & $613.3^{\mathrm{bc}}$ & $51.69 \mathrm{a}$ & $106.73^{\mathrm{a}}$ & $9.93^{\mathrm{a}}$ & $650.0^{\mathrm{a}}$ & $61.02^{\mathrm{a}}$ \\
\hline \multirow[t]{4}{*}{80} & 24 & & $89.77^{d}$ & $8.93^{\mathrm{de}}$ & $462.5^{\mathrm{f}}$ & $35.80^{\mathrm{d}}$ & $93.60^{\mathrm{e}}$ & $8.60^{\mathrm{a}}$ & $432.0^{\mathrm{g}}$ & $26.63^{\mathrm{h}}$ \\
\hline & 48 & & $95.40^{\mathrm{c}}$ & $9.17^{\mathrm{cd}}$ & $584.3^{\mathrm{cd}}$ & $36.86^{\mathrm{d}}$ & $96.00^{\mathrm{d}}$ & $9.23^{\mathrm{a}}$ & $561.5^{\mathrm{d}}$ & $37.50^{\mathrm{f}}$ \\
\hline & 72 & & $97.97^{\mathrm{b}}$ & $9.80^{\mathrm{a}}$ & $704.0^{\mathrm{a}}$ & $43.98^{c}$ & $98.73^{c}$ & $9.77^{\mathrm{a}}$ & $598.6^{\mathrm{c}}$ & $48.03^{\mathrm{d}}$ \\
\hline & 96 & & $96.97^{\mathrm{bc}}$ & $9.83^{\mathrm{a}}$ & $629.5^{\mathrm{b}}$ & $49.14^{\mathrm{b}}$ & $102.30^{\mathrm{b}}$ & $9.97^{\mathrm{a}}$ & $625.7^{b}$ & $56.33^{\mathrm{b}}$ \\
\hline \multirow[t]{4}{*}{60} & 24 & & $70.77^{\mathrm{g}}$ & $7.60^{\mathrm{h}}$ & $210.9^{\mathrm{i}}$ & $15.80^{\mathrm{g}}$ & $74.80^{\mathrm{i}}$ & $7.67^{\mathrm{a}}$ & $250.2^{\mathrm{j}}$ & $16.08^{\mathrm{j}}$ \\
\hline & 48 & & $82.47^{\mathrm{f}}$ & $8.33^{\mathrm{fg}}$ & $333.3^{\mathrm{h}}$ & $20.91^{\mathrm{f}}$ & $84.37^{\mathrm{h}}$ & $8.27^{\mathrm{a}}$ & $296.2^{\mathrm{i}}$ & $22.75^{\mathrm{i}}$ \\
\hline & 72 & & $81.83^{\mathrm{f}}$ & $7.97^{\text {gh }}$ & $340.1^{\mathrm{h}}$ & $20.71^{\mathrm{f}}$ & $95.10^{\mathrm{de}}$ & $8.33^{\mathrm{a}}$ & $374.7^{\mathrm{h}}$ & $27.85^{\mathrm{h}}$ \\
\hline & 96 & & $85.83^{\mathrm{e}}$ & $9.00^{\mathrm{de}}$ & $506.2^{\mathrm{e}}$ & $34.95^{\mathrm{d}}$ & $104.07^{\mathrm{b}}$ & $8.93^{\mathrm{a}}$ & $520.8^{\mathrm{e}}$ & $36.91^{\mathrm{f}}$ \\
\hline \multirow[t]{2}{*}{100} & & B. & $94.48^{\mathrm{b}}$ & $9.23^{\mathrm{a}}$ & $516.1^{\mathrm{d}}$ & $41.65^{\mathrm{c}}$ & $25.84^{\mathrm{a}}$ & $9.25^{\mathrm{a}}$ & $564.6^{\mathrm{b}}$ & $45.41^{\mathrm{a}}$ \\
\hline & & R.S. & $95.75^{\mathrm{a}}$ & $9.57^{\mathrm{a}}$ & $600.2^{\mathrm{b}}$ & $49.42^{\mathrm{a}}$ & $26.65^{\mathrm{a}}$ & $9.57^{\mathrm{a}}$ & $594.5^{\mathrm{a}}$ & $51.62^{\mathrm{a}}$ \\
\hline \multirow[t]{2}{*}{80} & & B. & $94.43^{\mathrm{b}}$ & $9.32^{\mathrm{a}}$ & $553.5^{\mathrm{c}}$ & $35.86^{\mathrm{d}}$ & $23.15 \mathrm{a}$ & $9.32^{\mathrm{a}}$ & $536.6^{c}$ & $39.43^{a}$ \\
\hline & & R.S. & $95.62^{\mathrm{ab}}$ & $9.55^{\mathrm{a}}$ & $636.6^{\mathrm{a}}$ & $47.03^{b}$ & $25.01^{\mathrm{a}}$ & $9.47^{\mathrm{a}}$ & $572.3^{\mathrm{b}}$ & $44.81^{\mathrm{a}}$ \\
\hline \multirow[t]{10}{*}{60} & & B. & $78.42^{\mathrm{d}}$ & $7.97^{\mathrm{a}}$ & $340.0^{\mathrm{e}}$ & $20.32^{\mathrm{f}}$ & $16.48^{\mathrm{a}}$ & $8.13^{\mathrm{a}}$ & $331.8^{\mathrm{e}}$ & $23.31^{\mathrm{a}}$ \\
\hline & & R.S. & $82.03^{c}$ & $8.48^{\mathrm{a}}$ & $355.2^{\mathrm{e}}$ & $25.86^{\mathrm{e}}$ & $18.21^{\mathrm{a}}$ & $8.47^{\mathrm{a}}$ & $389.2^{\mathrm{d}}$ & $28.48^{\mathrm{a}}$ \\
\hline & 24 & B. & $80.93^{a}$ & $8.13^{\mathrm{a}}$ & $327.3^{\mathrm{a}}$ & $21.19^{f}$ & $82.82^{\mathrm{a}}$ & $8.18^{\mathrm{a}}$ & $364.5^{\mathrm{a}}$ & $22.99^{\mathrm{h}}$ \\
\hline & & R.S. & $82.69^{a}$ & $8.67^{\mathrm{a}}$ & $399.3^{\mathrm{a}}$ & $32.76^{\mathrm{e}}$ & $85.69^{\mathrm{a}}$ & $8.56^{\mathrm{a}}$ & $411.8^{\mathrm{a}}$ & $28.09^{\mathrm{g}}$ \\
\hline & 48 & B. & $89.82^{\mathrm{a}}$ & $8.73^{\mathrm{a}}$ & $451.6^{\mathrm{a}}$ & $33.35^{\mathrm{de}}$ & $90.69^{\mathrm{a}}$ & $8.62^{\mathrm{a}}$ & $447.4^{\mathrm{a}}$ & $31.41^{\mathrm{f}}$ \\
\hline & & R.S. & $92.00^{\mathrm{a}}$ & $9.20^{\mathrm{a}}$ & $536.1^{\mathrm{a}}$ & $38.35^{\mathrm{c}}$ & $93.71^{\mathrm{a}}$ & $9.16^{\mathrm{a}}$ & $493.5^{\mathrm{a}}$ & $39.28^{\mathrm{e}}$ \\
\hline & 72 & B. & $92.56^{\mathrm{a}}$ & $9.04^{\mathrm{a}}$ & $536.3^{\mathrm{a}}$ & $35.03^{\mathrm{d}}$ & $95.27^{\mathrm{a}}$ & $9.27^{\mathrm{a}}$ & $517.1^{\mathrm{a}}$ & $41.08^{\mathrm{d}}$ \\
\hline & & R.S. & $95.04^{\mathrm{a}}$ & $9.24^{\mathrm{a}}$ & $584.7^{\mathrm{a}}$ & $42.32^{\mathrm{b}}$ & $98.38^{\mathrm{a}}$ & $9.27^{\mathrm{a}}$ & $552.6^{\mathrm{a}}$ & $45.06^{\mathrm{c}}$ \\
\hline & 96 & B. & $93.13^{\mathrm{a}}$ & $9.44^{\mathrm{a}}$ & $564.4^{\mathrm{a}}$ & $40.88^{b}$ & $98.11^{\mathrm{a}}$ & $9.53^{\mathrm{a}}$ & $580.8^{\mathrm{a}}$ & $48.72^{b}$ \\
\hline & & R.S. & $94.80^{\mathrm{a}}$ & $9.69^{\mathrm{a}}$ & $601.6^{\mathrm{a}}$ & $49.65^{\mathrm{a}}$ & $101.56^{\mathrm{a}}$ & $9.69^{\mathrm{a}}$ & $616.9^{\mathrm{a}}$ & $54.13^{\mathrm{a}}$ \\
\hline
\end{tabular}

- Values marked with the same letter(s) within the $1^{\text {st }}$ interaction effects are statistically similar using Revised LSD. Test at probability $=0.05$. Lowercase refer to the $1^{\text {st }}$ interaction effect. $\mathrm{I}=$ irrigation $\left(\% \mathrm{ET}_{\mathrm{C}}\right) \cdot \mathrm{K}=$ potassium $\left(\mathrm{K}_{2} \mathrm{O}\right.$ fed $\left.{ }^{-1}\right) \cdot \mathrm{M}=$ mulch type $، \mathrm{~B}=$ bare soil $، \mathrm{R} . \mathrm{S}$. = rice straw mulch

The interaction of three studied factors on plant height and dry weight plant ${ }^{-1}$, in both season, and

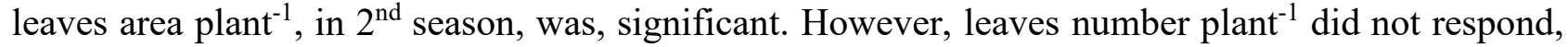
in both seasons. Generally, the interaction of irrigation $100 \% \mathrm{ET}_{\mathrm{C}} \times$ potassium at $72 \mathrm{and} / \mathrm{or} 96 \mathrm{~kg}$ $\mathrm{K}_{2} \mathrm{O}$ fed $^{-1} \times$ rice straw mulch produced taller stature, heaviest dry weight and larger leaves area plant $^{-1}$ than other combined treatments. 
Table 3. The $2^{\text {nd }}$ interaction of irrigation percent, potassium rate and mulch type on plant height $(\mathrm{cm})$, leaves number, leaves area $\left(\mathrm{cm}^{2}\right)$ and dry weight plant ${ }^{-1}(\mathrm{~g})$ during the winter seasons of 2016/2017 and $2017 / 2018$

\begin{tabular}{|c|c|c|c|c|c|c|c|c|c|c|}
\hline \multicolumn{3}{|c|}{ Treatments } & \multicolumn{4}{|l|}{2016} & \multicolumn{4}{|l|}{2017} \\
\hline I & $\mathbf{K}$ & $\mathbf{M}$ & $\begin{array}{l}\text { Plant } \\
\text { height }\end{array}$ & $\begin{array}{l}\text { leaves } \\
\text { number } \\
\text { plant }^{-1}\end{array}$ & $\begin{array}{l}\text { leaves } \\
\text { area } \\
\text { plant }^{-1}\end{array}$ & $\begin{array}{l}\text { dry } \\
\text { weight } \\
\text { plant }^{-1}\end{array}$ & $\begin{array}{l}\text { Plant } \\
\text { height }\end{array}$ & $\begin{array}{l}\text { leaves } \\
\text { number } \\
\text { plant }^{-1}\end{array}$ & $\begin{array}{l}\text { leaves } \\
\text { area } \\
\text { plant }^{-1}\end{array}$ & $\begin{array}{l}\text { Dry } \\
\text { weight } \\
\text { plant }^{-1}\end{array}$ \\
\hline \multirow[t]{8}{*}{100} & 24 & B & $84.80^{\mathrm{h}_{\mathrm{i}}}$ & $8.40^{\mathrm{a}}$ & $357.0^{\mathrm{a}}$ & $23.39^{k}$ & $83.00^{\circ}$ & $8.73^{\mathrm{a}}$ & $478.1^{\mathrm{h}}$ & $30.52^{\mathrm{k}}$ \\
\hline & & R.S. & $85.00^{\mathrm{hi}}$ & $8.93^{\mathrm{a}}$ & $475.9^{\mathrm{a}}$ & $35.27^{\mathrm{h}}$ & $85.73^{\mathrm{mno}}$ & $8.93^{\mathrm{a}}$ & $486.1^{\mathrm{h}}$ & $37.33^{\mathrm{i}}$ \\
\hline & 48 & B. & $93.60^{\mathrm{e}}$ & $9.13^{\mathrm{a}}$ & $521.3^{\mathrm{a}}$ & $48.32^{\mathrm{bc}}$ & $92.20^{\mathrm{jk}}$ & $8.80^{\mathrm{a}}$ & $524.4^{\mathrm{g}}$ & $41.31^{\mathrm{g}}$ \\
\hline & & R.S. & $96.13^{\text {cd }}$ & $9.67^{\mathrm{a}}$ & $609.5^{\mathrm{a}}$ & $51.24^{\mathrm{b}}$ & $98.00^{\text {efg }}$ & $9.53^{\mathrm{a}}$ & $582.6^{\mathrm{de}}$ & $50.27^{\mathrm{e}}$ \\
\hline & 72 & B & $99.33^{b}$ & $9.67^{\mathrm{a}}$ & $603.3^{a}$ & $46.59^{\mathrm{cd}}$ & $102.33^{\mathrm{cd}}$ & $9.67^{\mathrm{a}}$ & $613.4^{\mathrm{bc}}$ & $50.95^{\mathrm{e}}$ \\
\hline & & R.S. & $103.87^{\mathrm{a}}$ & $9.67^{\mathrm{a}}$ & $671.4^{\mathrm{a}}$ & $56.08^{\mathrm{a}}$ & $105.80^{\mathrm{ab}}$ & $9.73^{\mathrm{a}}$ & $651.6^{\mathrm{a}}$ & $55.69^{c}$ \\
\hline & 96 & B. & $100.20^{\mathrm{b}}$ & $9.73^{\mathrm{a}}$ & $582.9^{\mathrm{a}}$ & $48.31^{\mathrm{bc}}$ & $104.47^{\mathrm{bc}}$ & $9.80^{\mathrm{a}}$ & $642.3^{\mathrm{ab}}$ & $58.85^{\mathrm{b}}$ \\
\hline & & R.S. & $98.00^{\mathrm{bc}}$ & $10.00^{\mathrm{a}}$ & $643.9^{\mathrm{a}}$ & $55.08^{\mathrm{a}}$ & $109.00^{\mathrm{a}}$ & $10.07^{\mathrm{a}}$ & $657.7^{\mathrm{a}}$ & $63.19^{\mathrm{a}}$ \\
\hline \multirow[t]{8}{*}{80} & 24 & B & $91.00^{\mathrm{f}}$ & $8.60^{\mathrm{a}}$ & $437.8^{\mathrm{a}}$ & $27.38^{\mathrm{j}}$ & $93.87^{\mathrm{hij}}$ & $8.33^{\mathrm{a}}$ & $395.3^{\mathrm{i}}$ & $25.23^{\circ}$ \\
\hline & & R.S. & $88.53^{\mathrm{g}}$ & $9.27^{\mathrm{a}}$ & $487.1^{\mathrm{a}}$ & $44.22^{\mathrm{de}}$ & $93.33^{\mathrm{ijk}}$ & $8.87^{\mathrm{a}}$ & $468.7^{\mathrm{h}}$ & $28.02^{1 \mathrm{~m}}$ \\
\hline & 48 & B. & $95.13^{\mathrm{de}}$ & $9.00^{\mathrm{a}}$ & $514.0^{\mathrm{a}}$ & $33.60^{\mathrm{hi}}$ & $95.67^{\text {ghi }}$ & $9.07^{\mathrm{a}}$ & $546.3^{\mathrm{g}}$ & $33.77^{\mathrm{j}}$ \\
\hline & & R.S. & $95.67^{\text {cde }}$ & $9.33^{\mathrm{a}}$ & $654.5^{\mathrm{a}}$ & $40.11^{\mathrm{fg}}$ & $96.33^{\text {fghi }}$ & $9.40^{\mathrm{a}}$ & $576.8^{\text {ef }}$ & $41.22^{\mathrm{gh}}$ \\
\hline & 72 & B & $97.87^{\mathrm{bc}}$ & $9.87^{\mathrm{a}}$ & $668.1^{\mathrm{a}}$ & $39.27^{\mathrm{g}}$ & $96.80^{\text {fgh }}$ & $9.93^{\mathrm{a}}$ & $594.6^{\text {cde }}$ & $45.40^{\mathrm{f}}$ \\
\hline & & R.S. & $98.07^{\mathrm{bc}}$ & $9.73^{\mathrm{a}}$ & $739.1^{\mathrm{a}}$ & $48.69^{b c}$ & $100.67^{\mathrm{de}}$ & $9.60^{\mathrm{a}}$ & $602.6^{\text {cde }}$ & $50.66^{\mathrm{e}}$ \\
\hline & 96 & B. & $93.73^{\mathrm{de}}$ & $9.80^{\mathrm{a}}$ & $594.2^{\mathrm{a}}$ & $43.20^{\mathrm{ef}}$ & $99.47^{\mathrm{def}}$ & $9.93^{\mathrm{a}}$ & $610.2^{\text {cd }}$ & $53.32^{\mathrm{d}}$ \\
\hline & & R.S. & $100.20^{\mathrm{b}}$ & $9.87^{\mathrm{a}}$ & $664.7^{\mathrm{a}}$ & $55.09^{\mathrm{a}}$ & $105.13^{\mathrm{bc}}$ & $10.00^{\mathrm{a}}$ & $641.1^{\mathrm{ab}}$ & $59.35^{\mathrm{b}}$ \\
\hline \multirow[t]{8}{*}{60} & 24 & B. & $67.00^{1}$ & $7.40^{\mathrm{a}}$ & $186.9^{\mathrm{a}}$ & $12.80^{\mathrm{n}}$ & $71.60^{9}$ & $7.47^{\mathrm{a}}$ & $220.0^{1}$ & $13.23^{\mathrm{q}}$ \\
\hline & & R.S. & $74.53^{\mathrm{k}}$ & $7.80^{\mathrm{a}}$ & $234.1^{\mathrm{a}}$ & $18.79^{\mathrm{m}}$ & $78.00^{\mathrm{p}}$ & $7.87^{\mathrm{a}}$ & $280.5^{\mathrm{k}}$ & $18.93^{p}$ \\
\hline & 48 & B. & $80.73^{\mathrm{j}}$ & $8.07^{\mathrm{a}}$ & $319.6^{\mathrm{a}}$ & $18.12^{\mathrm{m}}$ & $84.20^{\text {no }}$ & $8.00^{\mathrm{a}}$ & $271.4^{\mathrm{k}}$ & $19.16^{\mathrm{p}}$ \\
\hline & & R.S. & $84.20^{\mathrm{hi}}$ & $8.60^{\mathrm{a}}$ & $346.9^{\mathrm{a}}$ & $23.70^{\mathrm{k}}$ & $86.80^{\mathrm{mn}}$ & $8.53^{\mathrm{a}}$ & $321.0^{j}$ & $26.34^{\text {no }}$ \\
\hline & 72 & B. & $80.47^{\mathrm{j}}$ & $7.60^{\mathrm{a}}$ & $337.4^{\mathrm{a}}$ & $19.23^{\mathrm{lm}}$ & $86.67^{\mathrm{mn}}$ & $8.20^{\mathrm{a}}$ & $345.9^{j}$ & $26.88^{\mathrm{mn}}$ \\
\hline & & R.S. & $83.20^{\mathrm{i}}$ & $8.33^{\mathrm{a}}$ & $342.7^{\mathrm{a}}$ & $22.19^{\mathrm{kl}}$ & $88.67^{\mathrm{lm}}$ & $8.47^{\mathrm{a}}$ & $403.6^{\mathrm{i}}$ & $28.82^{1}$ \\
\hline & 96 & B. & $85.47^{\mathrm{hi}}$ & $8.80^{\mathrm{a}}$ & $516.2^{\mathrm{a}}$ & $31.13^{\mathrm{i}}$ & $90.4^{0 \mathrm{kl}}$ & $8.87^{\mathrm{a}}$ & $489.8^{\mathrm{h}}$ & $33.98^{\mathrm{j}}$ \\
\hline & & R.S. & $86.20^{\text {gh }}$ & $9.20^{\mathrm{a}}$ & $496.3^{\mathrm{a}}$ & $38.77^{\mathrm{g}}$ & $90.53^{\mathrm{kl}}$ & $9.00^{\mathrm{a}}$ & $551.7^{\mathrm{fg}}$ & $39.84^{\mathrm{h}}$ \\
\hline
\end{tabular}

- Values marked with the same letter(s) within the $1^{\text {st }}$ interaction effects are statistically similar using Revised LSD. Test at probability $=0.05$. Lowercase refer to the $1^{\text {st }}$ interaction effect. $\mathrm{I}=$ irrigation $\left(\% \mathrm{ET}_{\mathrm{C}}\right) \cdot \mathrm{K}=$ potassium $\left(\mathrm{K}_{2} \mathrm{O}\right.$ fed $\left.{ }^{-1}\right) \cdot \mathrm{M}=$ mulch type $\cdot \mathrm{B}=$ bare soil $\cdot \mathrm{R} . \mathrm{S}$. = rice straw mulch

\section{Bulbs yield and its components}

The general effects (irrigation, potassium and mulch) of total bulbs yield and its components listed in Table 4 showed that were significant, in two investigated seasons.

Increasing the amount of irrigation at 80 and/or $100 \% \mathrm{ET}_{\mathrm{C}}$ was accompanied by increases in individual bulb weight, cloves weight and number bulb ${ }^{-1}$ and mean clove weight, in $1^{\text {st }}$ season, over irrigation amount at $60 \% \mathrm{ET}_{\mathrm{C}}$ while, increasing the amount of irrigation from 60 to 80 and further to $100 \% \mathrm{ET}_{\mathrm{C}}$ increases in individual bulb weight, cloves weight and number bulb ${ }^{-1}$, in $2^{\text {nd }}$ season. Therefore, increasing the amount of irrigation up $100 \% \mathrm{ET}_{\mathrm{C}}$ progressively increased total bulbs yield. The increasing application of potassium to grown plants from 24 to 48 and further to 72 and $96 \mathrm{~K}_{2} \mathrm{O}$ fed $^{-1}$ gave, significant, gradual increases in total bulbs yield and its components. The exception was in cloves number bulb ${ }^{-1}$ where the difference potassium rate at 48 and $72 \mathrm{~K}_{2} \mathrm{O}$ fed $^{-1}$ was at par. The effect noticed from comparisons between two mulch types reflected that, rice straw mulch, significantly, increased total bulbs yield and its components over bare soil.

The influence of interaction between irrigation and potassium levels on total bulbs yield and its components was significant, in both seasons except cloves number bulb ${ }^{-1}$, in $2^{\text {nd }}$ season (Table 5).

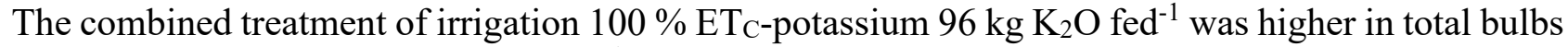
yield, blub weight, cloves weight bulb ${ }^{-1}$ and clove weight than the other combinations. However, the 


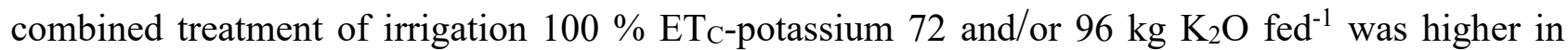
cloves number bulb ${ }^{-1}$ than the other combinations.

The impact of interaction between irrigation and mulch levels on bulb weight and cloves weight bulb $^{-1}$ was significant however, total bulbs yield was not responded, in both seasons. Meanwhile, clove weight and cloves number bulb ${ }^{-1}$ were significant in $1^{\text {st }}$ and $2^{\text {nd }}$ season, orderly (Table 5). The combination of irrigation 80 and/or $100 \%$ ET $_{C}$ and rice straw mulch seemed to be the highest mean values in a single bulb weight, cloves weight and number bulb ${ }^{-1}$ whereas, irrigation $80 \% \mathrm{ET}_{\mathrm{C}}$ and rice straw mulch was the best in clove weight.

The effect of interaction between potassium and mulch on total bulbs yield and bulb weight, in both seasons and cloves weight, number bulb ${ }^{-1}$ and clove weight, in $1^{\text {st }}$ season was significant (Table 5). The combination of potassium $96 \mathrm{~kg} \mathrm{~K}_{2} \mathrm{O} \mathrm{fed}^{-1}$-rice straw mulch had the maximum values in total bulbs yield, bulb weight, cloves weight and number bulb ${ }^{-1}$. Cloves number bulb ${ }^{-1}$ was the best as combined treatment at potassium $48 \mathrm{~K}_{2} \mathrm{O}$ fed $^{-1}$ with rice straw mulch.

The $2^{\text {nd }}$ order interaction among three factors on total bulbs yield and clove weight was significant, in both seasons while, bulb weight and cloves weight bulb- 1 was, truly, in $2^{\text {nd }}$ season only meanwhile, cloves number bulb ${ }^{-1}$ was, intrinsically, in $1^{\text {st }}$ season only. The combined treatment to grown plants; irrigation $100 \% \mathrm{ET}_{\mathrm{C}}$ and application potassium $96 \mathrm{~kg} \mathrm{~K}_{2} \mathrm{O} \mathrm{fed}^{-1}$ as well as covering soil surface with rice straw attained higher, total bulbs yield, bulb weight, cloves weight bulb ${ }^{-1}$ and clove weight than the other combinations. Mean value of cloves number bulb ${ }^{-1}$ was the maximum when garlic plants irrigated with $100 \% \mathrm{ET}_{\mathrm{C}}$ and fertilized with $72 \mathrm{~kg} \mathrm{K2O}$ in addition to surface with rice straw mulch.

Table 4. The main effect of irrigation percent, potassium rate and mulch type on total bulbs yield (tones), bulb weight $(\mathrm{g})$, clove weight plant $^{-1}(\mathrm{~g})$, cloves number plant ${ }^{-1}(\mathrm{~g})$ and mean clove weight (g), during the winter seasons of 2016 and 2017

\begin{tabular}{|c|c|c|c|c|c|c|c|c|c|c|}
\hline \multirow{2}{*}{$\begin{array}{l}\text { Treatments } \\
\text { Irrigation } \\
\text { (\% ETC) }\end{array}$} & \multicolumn{5}{|c|}{2016} & \multicolumn{5}{|c|}{2017} \\
\hline & $\begin{array}{l}\text { Total } \\
\text { bulbs } \\
\text { yield }\end{array}$ & $\begin{array}{l}\text { Bulb } \\
\text { weight }\end{array}$ & $\begin{array}{l}\text { Cloves } \\
\text { weight } \\
\text { bulb }^{-1}\end{array}$ & $\begin{array}{l}\text { Cloves } \\
\text { No. } \\
\text { bulb }^{-1}\end{array}$ & $\begin{array}{l}\text { Mean } \\
\text { clove } \\
\text { weight }\end{array}$ & $\begin{array}{l}\text { Total } \\
\text { bulbs } \\
\text { yield }\end{array}$ & $\begin{array}{l}\text { Bulb } \\
\text { weight }\end{array}$ & $\begin{array}{l}\text { Cloves } \\
\text { weight } \\
\text { bulb }^{-1}\end{array}$ & $\begin{array}{l}\text { Cloves } \\
\text { No. } \\
\text { bulb }^{-1}\end{array}$ & $\begin{array}{l}\text { Mean } \\
\text { Clove } \\
\text { weight }\end{array}$ \\
\hline 100 & $8.02^{\mathrm{A}}$ & $94.58^{\mathrm{A}}$ & $83.75^{\mathrm{A}}$ & $16.51^{\mathrm{A}}$ & $5.04^{\mathrm{A}}$ & $8.25^{\mathrm{A}}$ & $97.05^{\mathrm{A}}$ & $90.18^{\mathrm{A}}$ & $16.95^{\mathrm{A}}$ & $5.29^{\mathrm{B}}$ \\
\hline 80 & $7.38^{\mathrm{B}}$ & $90.10^{\mathrm{A}}$ & $80.40^{\mathrm{A}}$ & $15.20^{\mathrm{AB}}$ & $5.30^{\mathrm{A}}$ & $7.59^{\mathrm{B}}$ & $93.80^{\mathrm{B}}$ & $87.03^{\mathrm{B}}$ & $15.14^{\mathrm{B}}$ & $5.71^{\mathrm{A}}$ \\
\hline 60 & $5.42^{\mathrm{C}}$ & $56.26^{\mathrm{B}}$ & $51.67^{\mathrm{B}}$ & $14.75^{\mathrm{B}}$ & $3.46^{\mathrm{B}}$ & $5.60^{\mathrm{C}}$ & $59.58^{\mathrm{C}}$ & $55.33^{\mathrm{C}}$ & $14.16^{\mathrm{C}}$ & $3.85^{\mathrm{C}}$ \\
\hline \multicolumn{11}{|l|}{$\begin{array}{l}\text { Potassium } \\
\left(\text { K2O fed }^{-1}\right)\end{array}$} \\
\hline 24 & $6.28^{\mathrm{D}}$ & $54.08^{\mathrm{D}}$ & $48.99^{\mathrm{D}}$ & $14.58^{\mathrm{C}}$ & $3.31^{\mathrm{D}}$ & $6.43^{\mathrm{D}}$ & $58.99^{\mathrm{D}}$ & $54.15^{\mathrm{D}}$ & $13.71^{\mathrm{D}}$ & $3.93^{\mathrm{D}}$ \\
\hline 48 & $6.75^{\mathrm{C}}$ & $71.87^{\mathrm{C}}$ & $65.54^{\mathrm{C}}$ & $15.46^{\mathrm{B}}$ & $4.25^{\mathrm{C}}$ & $6.93^{\mathrm{C}}$ & $77.10^{\mathrm{C}}$ & $71.74^{C}$ & $15.04^{\mathrm{C}}$ & $4.75^{\mathrm{C}}$ \\
\hline 72 & $7.10^{\mathrm{B}}$ & $90.68^{\mathrm{B}}$ & $80.97^{\mathrm{B}}$ & $15.83^{\mathrm{AB}}$ & $5.13^{\mathrm{B}}$ & $7.40^{\mathrm{B}}$ & $90.36^{\mathrm{B}}$ & $83.94^{\mathrm{B}}$ & $16.21^{\mathrm{B}}$ & $5.14^{\mathrm{B}}$ \\
\hline 96 & $7.63^{\mathrm{A}}$ & $104.6^{\mathrm{A}}$ & $92.25^{\mathrm{A}}$ & $16.08^{\mathrm{A}}$ & $5.72^{\mathrm{A}}$ & $7.83^{\mathrm{A}}$ & $107.4^{\mathrm{A}}$ & $\underset{\mathrm{A}}{100.22}$ & $16.70^{\mathrm{A}}$ & $5.98^{\mathrm{A}}$ \\
\hline \multicolumn{11}{|c|}{ Mulch(type) } \\
\hline Bare soil & $6.83^{\mathrm{B}}$ & $73.74^{\mathrm{B}}$ & $66.20^{\mathrm{B}}$ & $14.94^{\mathrm{B}}$ & $4.38^{\mathrm{B}}$ & $6.99^{\text {в }}$ & $78.44^{\mathrm{B}}$ & $72.71^{\mathrm{B}}$ & $15.09^{\mathrm{B}}$ & $4.73^{\mathrm{B}}$ \\
\hline Rice straw & $7.05^{\mathrm{A}}$ & $86.89^{\mathrm{A}}$ & $77.68^{\mathrm{A}}$ & $16.03^{\mathrm{A}}$ & $4.82^{\mathrm{A}}$ & $7.31^{\mathrm{A}}$ & $88.51^{\mathrm{A}}$ & $82.32^{\mathrm{A}}$ & $15.74^{\mathrm{A}}$ & $5.17^{\mathrm{A}}$ \\
\hline
\end{tabular}

-Values marked with the same letter(s) within the main effect are statistically similar using Revised LSD. Test at probability $=0.05$. Uppercase refer to the main effect. 
Table 5. The $1^{\text {st }}$ interaction of irrigation percent, potassium rate and mulch type on total bulbs yield (tones), bulb weight (g), clove weight plant $^{-1}(\mathrm{~g})$, cloves number plant ${ }^{-1}(\mathrm{~g})$ and mean clove weight (g), during the winter seasons of 2016 and 2017

\begin{tabular}{|c|c|c|c|c|c|c|c|c|c|c|c|c|}
\hline \multicolumn{3}{|c|}{ Treatments } & \multicolumn{5}{|c|}{2016} & \multicolumn{5}{|c|}{2017} \\
\hline I. & $\mathbf{K}$. & M. & $\begin{array}{l}\text { Total } \\
\text { bulbs } \\
\text { yield }\end{array}$ & & $\begin{array}{l}\text { Cloves } \\
\text { weight } \\
\text { bulb }^{-1}\end{array}$ & $\begin{array}{l}\text { Cloves } \\
\text { No. } \\
\text { bulb }^{-1}\end{array}$ & $\begin{array}{l}\text { Mean } \\
\text { clove } \\
\text { weight }\end{array}$ & $\begin{array}{l}\text { Total } \\
\text { bulbs } \\
\text { yield }\end{array}$ & & $\begin{array}{l}\text { Cloves } \\
\text { weight } \\
\text { bulb }^{-1}\end{array}$ & $\begin{array}{l}\text { Cloves } \\
\text { No. } \\
\text { bulb }^{-1}\end{array}$ & $\begin{array}{l}\text { Mean } \\
\text { Clove } \\
\text { weight }\end{array}$ \\
\hline \multirow[t]{4}{*}{100} & 24 & & & & & & $3.67^{\mathrm{e}}$ & & & & $15.13^{\mathrm{a}}$ & $4.19^{\mathrm{e}}$ \\
\hline & 48 & & $8.06^{\mathrm{bc}}$ & $.54^{\mathrm{e}}$ & $76.41^{d}$ & 15.8 & $4.82^{\mathrm{c}}$ & 0.14 & 5 & $1.66^{\mathrm{e}}$ & $16.63^{a}$ & $4.93^{\mathrm{d}}$ \\
\hline & 72 & & $8.19^{\mathrm{ab}}$ & $105.51^{\mathrm{c}}$ & $94.46^{\mathrm{b}}$ & $17.47^{\mathrm{a}}$ & 3.4 & $p$ & $104.84^{\mathrm{c}}$ & $97.63^{\mathrm{c}}$ & $17.80^{\mathrm{a}}$ & $5.49^{\mathrm{c}}$ \\
\hline & 96 & & $8.33^{\mathrm{a}}$ & $125.36^{\mathrm{a}}$ & $107.05^{\mathrm{a}}$ & $17.17^{\mathrm{a}}$ & $6.24^{\mathrm{a}}$ & $8.64 \mathrm{a}$ & $126.36^{\mathrm{a}}$ & $117.9^{\mathrm{a}}$ & $18.23^{\mathrm{a}}$ & $6.54 \mathrm{a}$ \\
\hline \multirow[t]{4}{*}{80} & 24 & & $6.73^{\mathrm{f}}$ & $69.09^{\mathrm{g}}$ & $61.33^{\mathrm{f}}$ & $15.00^{\mathrm{bc}}$ & $4.09^{d}$ & $6.73^{g}$ & $72.19^{\mathrm{g}}$ & $66.42^{\mathrm{g}}$ & $13.23^{\mathrm{a}}$ & $5.04^{\mathrm{d}}$ \\
\hline & 48 & & $7.26^{\mathrm{e}}$ & $79.05^{f}$ & $72.56^{\mathrm{de}}$ & $15.43^{\mathrm{bc}}$ & $4.75^{\mathrm{c}}$ & $7.57^{f}$ & $87.28^{\mathrm{e}}$ & $81.46^{\mathrm{e}}$ & $14.60^{\mathrm{a}}$ & $5.59^{\mathrm{bc}}$ \\
\hline & 72 & & $7.51 \mathrm{~d}$ & $100.69^{d}$ & $89.31^{\mathrm{c}}$ & $14.57^{\mathrm{c}}$ & $6.13^{\mathrm{a}}$ & $7.89^{\mathrm{d}}$ & $98.64^{\mathrm{d}}$ & $91.67^{\mathrm{d}}$ & $16.00^{\mathrm{a}}$ & $5.73^{b}$ \\
\hline & 96 & & $8.03^{\circ}$ & $111.57^{\mathrm{b}}$ & $98.40^{\mathrm{b}}$ & $15.80^{\mathrm{b}}$ & $6.24^{\mathrm{a}}$ & $8.18^{\mathrm{c}}$ & $117.07^{\mathrm{b}}$ & $108.5^{\mathrm{b}}$ & $16.73^{\mathrm{a}}$ & $6.49 \mathrm{a}$ \\
\hline \multirow[t]{4}{*}{60} & 24 & & $4.61^{\mathrm{j}}$ & $31.24^{\mathrm{j}}$ & $28.58^{\mathrm{h}}$ & $13.20^{\mathrm{d}}$ & $2.15^{\mathrm{g}}$ & $4.78^{j}$ & $35.85^{\mathrm{j}}$ & $32.51^{\mathrm{j}}$ & $12.77^{\mathrm{a}}$ & $2.55^{\mathrm{g}}$ \\
\hline & 48 & & $4.95^{\mathrm{i}}$ & $51.02^{\mathrm{i}}$ & $47.64^{\mathrm{g}}$ & $15.07^{\mathrm{bc}}$ & $3.16^{\mathrm{f}}$ & $5.09^{1}$ & $55.93^{\mathrm{i}}$ & $52.10^{\mathrm{i}}$ & $13.90^{\mathrm{a}}$ & $3.74^{\mathrm{f}}$ \\
\hline & 72 & & $5.61^{\mathrm{h}}$ & $65.85^{\mathrm{gh}}$ & $59.14^{\mathrm{f}}$ & $15.47^{\mathrm{bc}}$ & $3.84^{\mathrm{de}}$ & $5.88^{\mathrm{h}}$ & $67.60^{\mathrm{h}}$ & $62.51^{\mathrm{h}}$ & $14.83^{\mathrm{a}}$ & $4.22^{\mathrm{e}}$ \\
\hline & 96 & & $6.51^{\mathrm{g}}$ & $76.93^{\mathrm{f}}$ & $71.31^{\mathrm{e}}$ & $15.27^{\mathrm{bc}}$ & $4.68^{\mathrm{c}}$ & $6.66^{\mathrm{g}}$ & $78.93^{\mathrm{f}}$ & $74.19^{f}$ & $15.13^{\mathrm{a}}$ & $4.91^{\mathrm{d}}$ \\
\hline \multirow[t]{2}{*}{100} & & B. & $7.91^{\mathrm{a}}$ & $87.67^{c}$ & $78.38^{\mathrm{b}}$ & $15.93^{b}$ & $4.86^{\mathrm{a}}$ & $8.11^{\mathrm{a}}$ & $92.18^{b}$ & $85.38^{\mathrm{b}}$ & $16.45^{\mathrm{a}}$ & $5.14^{\mathrm{c}}$ \\
\hline & & $\mathbf{R}$ & $8.13^{\mathrm{a}}$ & $101.49^{\mathrm{a}}$ & $89.12^{\mathrm{a}}$ & $17.08^{\mathrm{a}}$ & $5.21^{\mathrm{a}}$ & $8.39^{\mathrm{a}}$ & $101.92^{\mathrm{a}}$ & $94.99^{\mathrm{a}}$ & $17.45^{\mathrm{a}}$ & $5.44^{\mathrm{b}}$ \\
\hline \multirow[t]{2}{*}{80} & & B. & $7.27^{\mathrm{a}}$ & $82.11^{\mathrm{d}}$ & $73.42^{\mathrm{c}}$ & $14.28^{\mathrm{d}}$ & $5.12^{\mathrm{a}}$ & $7.45^{\mathrm{a}}$ & $87.14^{c}$ & $80.53^{\mathrm{c}}$ & $15.00^{\mathrm{a}}$ & $5.33^{b c}$ \\
\hline & & & $7.49^{\mathrm{a}}$ & $98.09^{\mathrm{b}}$ & $87.38^{a}$ & $16.12^{\mathrm{b}}$ & $5.49^{\mathrm{a}}$ & $7.74^{\mathrm{a}}$ & $100.45^{\mathrm{a}}$ & $93.52^{\mathrm{a}}$ & $15.28^{\mathrm{a}}$ & $6.09^{\mathrm{a}}$ \\
\hline \multirow[t]{10}{*}{60} & & $\mathbf{P}$ & $5.30^{\mathrm{a}}$ & $51.43^{\mathrm{f}}$ & $46.80^{\mathrm{e}}$ & $14.60^{\mathrm{cd}}$ & $3.14^{\mathrm{a}}$ & $5.41^{\mathrm{a}}$ & $56.00^{\mathrm{e}}$ & $52.21^{\mathrm{e}}$ & $13.83^{\mathrm{a}}$ & $3.72^{\mathrm{e}}$ \\
\hline & & $\mathbf{R}$ & $5.54^{\mathrm{a}}$ & $61.09^{\mathrm{e}}$ & $56.53^{\mathrm{d}}$ & $14.90^{\mathrm{c}}$ & $3.78^{\mathrm{a}}$ & $5.80^{\mathrm{a}}$ & $63.16^{d}$ & $58.45^{\mathrm{d}}$ & $14.48^{\mathrm{a}}$ & $3.99^{\mathrm{d}}$ \\
\hline & 24 & B. & $6.09^{f}$ & $46.73^{\mathrm{g}}$ & $42.18^{g}$ & $13.40^{\mathrm{d}}$ & $3.12^{\mathrm{f}}$ & $6.24^{\mathrm{h}}$ & $54.54^{\mathrm{h}}$ & $49.91^{\mathrm{a}}$ & $13.36^{\mathrm{a}}$ & $3.71^{\mathrm{a}}$ \\
\hline & & R.S & $6.46^{\mathrm{e}}$ & $61.43^{\mathrm{f}}$ & $55.81^{\mathrm{f}}$ & $15.76^{\mathrm{ab}}$ & $3.49^{\mathrm{e}}$ & $6.62^{\mathrm{g}}$ & $63.43^{\mathrm{g}}$ & $58.40^{\mathrm{a}}$ & $14.07^{\mathrm{a}}$ & $4.14^{\mathrm{a}}$ \\
\hline & 48 & B. & $6.69^{\mathrm{d}}$ & $66.98^{\mathrm{e}}$ & $61.42^{\mathrm{e}}$ & $14.56^{\mathrm{c}}$ & $4.25^{\mathrm{d}}$ & 6. & $71.44^{\mathrm{f}}$ & $66.41^{\mathrm{a}}$ & $14.49^{\mathrm{a}}$ & $4.55^{\mathrm{a}}$ \\
\hline & & R.S & $6.82^{\mathrm{d}}$ & $76.76^{\mathrm{d}}$ & $69.65^{\mathrm{d}}$ & $16.36^{\mathrm{a}}$ & $4.24^{\mathrm{d}}$ & 7 & $82.76^{\mathrm{e}}$ & $77.07^{\mathrm{a}}$ & $15.60^{\mathrm{a}}$ & $4.95^{\mathrm{a}}$ \\
\hline & 72 & B. & $7.05^{\mathrm{c}}$ & $84.95^{\mathrm{c}}$ & $75.56^{\mathrm{c}}$ & $15.62^{\mathrm{b}}$ & $4.86^{\mathrm{c}}$ & $7.31^{\mathrm{d}}$ & $86.36^{\mathrm{d}}$ & $79.90^{\mathrm{a}}$ & $16.02^{\mathrm{a}}$ & $4.94^{\mathrm{a}}$ \\
\hline & & R.S. & $7.16^{\mathrm{c}}$ & $96.41^{\mathrm{b}}$ & $86.38^{\mathrm{b}}$ & $16.04^{\mathrm{ab}}$ & $5.39^{\mathrm{b}}$ & $7.49^{\mathrm{c}}$ & $94.36^{\mathrm{c}}$ & $87.97^{\mathrm{a}}$ & $16.40^{\mathrm{a}}$ & $5.34^{\mathrm{a}}$ \\
\hline & 96 & B. & $7.47^{\mathrm{b}}$ & $96.29^{\mathrm{b}}$ & $85.65^{\mathrm{b}}$ & $16.18^{\mathrm{ab}}$ & $5.27^{\mathrm{b}}$ & $7.63^{b}$ & $101.42^{\mathrm{b}}$ & $94.61^{\mathrm{a}}$ & $16.51^{\mathrm{a}}$ & $5.70^{\mathrm{a}}$ \\
\hline & & R.S. & $7.78^{\mathrm{a}}$ & $112.95^{\mathrm{a}}$ & $98.86^{\mathrm{a}}$ & $15.98^{\mathrm{ab}}$ & $6.17^{\mathrm{a}}$ & $8.03^{\mathrm{a}}$ & $113.48^{\mathrm{a}}$ & $105.8^{\mathrm{a}}$ & $16.89^{\mathrm{a}}$ & $6.26^{\mathrm{a}}$ \\
\hline
\end{tabular}

- Values marked with the same letter(s) within the $1^{\text {st }}$ interaction effects are statistically similar using Revised LSD. Test at probability $=0.05$. Lowercase refer to the $1^{\text {st }}$ interaction effect. $\mathrm{I}=$ irrigation $\left(\% \mathrm{ET}_{\mathrm{C}}\right) \cdot \mathrm{K}=\operatorname{potassium}\left(\mathrm{K}_{2} \mathrm{O}\right.$ fed $\left.{ }^{-1}\right) \cdot \mathrm{M}=$ mulch type $، \mathrm{~B}=$ bare soil $\cdot \mathrm{R}$.S.= rice straw mulch 
Table 6. The $2^{\text {nd } t}$ interaction of irrigation percent, potassium rate and mulch type on total bulbs yield (tones), bulb weight (g), clove weight plant ${ }^{-1}(\mathrm{~g})$, cloves number plant $^{-1}(\mathrm{~g})$ and mean clove weight (g), during the winter seasons of 2016 and 2017

\begin{tabular}{|c|c|c|c|c|c|c|c|c|c|c|c|c|}
\hline \multicolumn{3}{|c|}{ Treatments } & \multicolumn{6}{|c|}{2016} & \multicolumn{4}{|c|}{2017} \\
\hline I & $\mathbf{K}$ & $\mathbf{M}$ & $\begin{array}{l}\text { Total } \\
\text { bulbs } \\
\text { yield }\end{array}$ & $\begin{array}{l}\text { Bulb } \\
\text { weight }\end{array}$ & $\begin{array}{l}\text { Cloves } \\
\text { weight } \\
\text { bulb }^{-1}\end{array}$ & $\begin{array}{l}\text { Cloves } \\
\text { No. } \\
\text { bulb }^{-1}\end{array}$ & $\begin{array}{l}\text { Mean } \\
\text { clove } \\
\text { weight }\end{array}$ & $\begin{array}{l}\text { Total } \\
\text { bulbs } \\
\text { yield }\end{array}$ & $\begin{array}{l}\text { ulb } \\
\text { eight }\end{array}$ & $\begin{array}{l}\text { Cloves } \\
\text { weight } \\
\text { bulb }^{-1}\end{array}$ & $\begin{array}{l}\text { Cloves } \\
\text { No. } \\
\text { bulb }^{-1}\end{array}$ & $\begin{array}{l}\text { Mean } \\
\text { Clove } \\
\text { weight }\end{array}$ \\
\hline \multirow[t]{8}{*}{100} & 24 & B. & & $55.23^{\mathrm{a}}$ & $.81^{\mathrm{a}}$ & & & & $5.35^{1}$ & 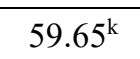 & $14.4^{\mathrm{a}}$ & \\
\hline & & R.S. & $7.76^{\mathrm{de}}$ & 68.5 & 63.3 & $16.80^{\mathrm{abc}}$ & $3.77^{\mathrm{hij}}$ & $8.00^{\mathrm{d}}$ & $72.49^{\mathrm{k}}$ & $7.42^{\mathrm{j}}$ & $15.8^{\mathrm{a}}$ & $4.25^{\mathrm{ij}}$ \\
\hline & 48 & B. & 8. & 8 & 73 & $15.33^{\text {defgh }}$ & $4.77 \mathrm{def}$ & $7.99^{d}$ & $81.89^{\mathrm{hi}}$ & $75.74^{\mathrm{i}}$ & $15.7^{\mathrm{a}}$ & $4.82^{\mathrm{gh}}$ \\
\hline & & R.S. & $8.09^{\mathrm{bc}}$ & $90.63^{\mathrm{a}}$ & $79.78^{a}$ & $16.40^{\text {bcd }}$ & $4.88^{\mathrm{def}}$ & $8.30^{\mathrm{c}}$ & $94.27^{\mathrm{f}}$ & $87.58^{f}$ & $17.5^{\mathrm{a}}$ & $5.03^{\mathrm{fg}}$ \\
\hline & 72 & B. & $8.13^{\mathrm{abc}}$ & $99.57^{\mathrm{a}}$ & $90.55^{\mathrm{a}}$ & $17.07^{\mathrm{ab}}$ & $5.30^{\mathrm{bcd}}$ & $8.40^{\mathrm{bc}}$ & $105.00^{\mathrm{e}}$ & $97.81^{\mathrm{e}}$ & $17.6^{\mathrm{a}}$ & $5.54^{\mathrm{de}}$ \\
\hline & & R.S. & $8.26^{\mathrm{ab}}$ & $111.45^{\mathrm{a}}$ & $98.37^{\mathrm{a}}$ & $17.87^{\mathrm{a}}$ & $5.52^{\mathrm{bc}}$ & $8.46^{\mathrm{bc}}$ & $104.67^{\mathrm{e}}$ & $97.44^{\mathrm{e}}$ & $17.9^{\mathrm{a}}$ & $5.43^{\mathrm{def}}$ \\
\hline & 96 & B. & $8.25^{\mathrm{ab}}$ & $115.42^{\mathrm{a}}$ & $99.12^{\mathrm{a}}$ & $17.07^{\mathrm{ab}}$ & $5.81^{\mathrm{b}}$ & $8.50^{\mathrm{b}}$ & $116.48^{\mathrm{c}}$ & $108.31^{\mathrm{c}}$ & $18.0^{\mathrm{a}}$ & $6.04^{\mathrm{bc}}$ \\
\hline & & R.S. & $8.42^{\mathrm{a}}$ & $135.30^{\mathrm{a}}$ & $114.98^{\mathrm{a}}$ & $17.27^{\mathrm{ab}}$ & $6.67^{\mathrm{a}}$ & $8.79^{\mathrm{a}}$ & $136.24^{\mathrm{a}}$ & $127.5^{\mathrm{a}}$ & $18.4^{\mathrm{a}}$ & $7.04^{\mathrm{a}}$ \\
\hline \multirow[t]{8}{*}{80} & 24 & B. & $6.47^{\mathrm{j}}$ & $59.74^{\mathrm{a}}$ & $53.37^{\mathrm{a}}$ & $13.33^{\mathrm{jk}}$ & $4.02^{\mathrm{ghi}}$ & $6.50^{\mathrm{h}}$ & $64.85^{1}$ & $59.32^{\mathrm{k}}$ & $13.1^{\mathrm{a}}$ & $4.53^{\mathrm{hi}}$ \\
\hline & & R.S. & $6.99^{\mathrm{h}} \mathrm{i}$ & $78.45^{a}$ & $69.30^{\mathrm{a}}$ & $16.67^{\mathrm{abc}}$ & $4.17^{\mathrm{gh}}$ & $6.97^{\mathrm{g}}$ & $79.53^{\mathrm{ij}}$ & $73.52^{\mathrm{i}}$ & $13.3^{\mathrm{a}}$ & $5.54^{\mathrm{de}}$ \\
\hline & 48 & B. & $7.22^{\mathrm{gh}}$ & $74.49^{\mathrm{a}}$ & $68.47^{\mathrm{a}}$ & $13.53^{\mathrm{jk}}$ & $5.08^{\mathrm{cd}}$ & $7.39^{f}$ & $83.86^{\mathrm{h}}$ & $77.74^{\mathrm{hi}}$ & $14.4^{\mathrm{a}}$ & $5.40^{\mathrm{def}}$ \\
\hline & & R.S. & $7.29^{\mathrm{fg}}$ & $83.60^{\mathrm{a}}$ & $76.64^{a}$ & $17.33^{a b}$ & $4.42^{\mathrm{efg}}$ & $7.76^{\mathrm{e}}$ & $90.69^{\mathrm{fg}}$ & $85.18^{\mathrm{fg}}$ & $14.8^{\mathrm{a}}$ & $5.77^{\mathrm{cd}}$ \\
\hline & 72 & B. & $7.49^{\mathrm{fg}}$ & $92.86^{\mathrm{a}}$ & $81.38^{\mathrm{a}}$ & $14.13^{\mathrm{hij}}$ & $5.78^{b}$ & $7.88^{\mathrm{de}}$ & $89.17^{\mathrm{g}}$ & $82.39^{\mathrm{gh}}$ & $15.8^{\mathrm{a}}$ & $5.22^{\mathrm{efg}}$ \\
\hline & & R.S. & $7.53^{\text {ef }}$ & $108.52^{\mathrm{a}}$ & $97.25^{\mathrm{a}}$ & $15.00^{\text {efgh }}$ & $6.48^{\mathrm{a}}$ & $7.89^{\mathrm{de}}$ & $108.12^{\mathrm{e}}$ & $100.9^{\mathrm{de}}$ & $16.2^{\mathrm{a}}$ & $6.24^{\mathrm{b}}$ \\
\hline & 96 & B. & $7.90^{\mathrm{cc}}$ & $101.37^{\mathrm{a}}$ & $90.48^{\mathrm{a}}$ & $16.13^{\text {bcde }}$ & $5.60^{b c}$ & $8.02^{\mathrm{d}}$ & $110.67^{\mathrm{d}}$ & $102.68^{\mathrm{d}}$ & $16.6^{\mathrm{a}}$ & $6.16^{\mathrm{bc}}$ \\
\hline & & R.S. & $8.16^{\mathrm{ab}}$ & $121.78^{a}$ & $106.32^{\mathrm{a}}$ & $15.47^{\text {defg }}$ & $6.88^{\mathrm{a}}$ & $8.35^{\mathrm{bc}}$ & $123.47^{\mathrm{b}}$ & $114.45^{\mathrm{b}}$ & $16.8^{\mathrm{a}}$ & $6.82^{\mathrm{a}}$ \\
\hline \multirow[t]{8}{*}{60} & 24 & B. & $4.59^{\mathrm{m}}$ & $25.23^{\mathrm{a}}$ & $22.37^{\mathrm{a}}$ & $12.60^{\mathrm{k}}$ & $1.78^{1}$ & $4.66^{\mathrm{m}}$ & $33.42^{\circ}$ & $30.76^{\mathrm{m}}$ & $12.5^{\mathrm{a}}$ & $2.46^{1}$ \\
\hline & & R.S. & $4.63^{\mathrm{m}}$ & $37.25^{\mathrm{a}}$ & $34.78^{\mathrm{a}}$ & $13.80^{\mathrm{ij}}$ & $2.52^{\mathrm{k}}$ & $4.89^{1}$ & $38.29^{\mathrm{n}}$ & $34.25^{\mathrm{m}}$ & $13.0^{\mathrm{a}}$ & $2.63^{1}$ \\
\hline & 48 & B. & $4.831^{\mathrm{m}}$ & $45.99^{\mathrm{a}}$ & $42.74^{\mathrm{a}}$ & $14.80^{\mathrm{fghi}}$ & $2.89^{\mathrm{k}}$ & $4.93^{1}$ & $48.55^{\mathrm{m}}$ & $45.75^{1}$ & $13.3^{\mathrm{a}}$ & $3.43^{\mathrm{k}}$ \\
\hline & & R.S. & $5.07^{1}$ & $56.06^{\mathrm{a}}$ & $52.54^{\mathrm{a}}$ & $15.33^{\text {defgh }}$ & $3.44^{\mathrm{j}}$ & $5.25^{\mathrm{k}}$ & $63.31^{1}$ & $58.46^{\mathrm{k}}$ & $14.4^{\mathrm{a}}$ & $4.05^{\mathrm{j}}$ \\
\hline & 72 & B. & $5.52^{\mathrm{k}}$ & $62.42^{\mathrm{a}}$ & $54.76^{\mathrm{a}}$ & $15.67^{\text {cdef }}$ & $3.50^{\mathrm{ij}}$ & $5.65^{\mathrm{j}}$ & $64.91^{1}$ & $59.49^{\mathrm{k}}$ & $14.6^{\mathrm{a}}$ & $4.07^{\mathrm{j}}$ \\
\hline & & R.S. & $5.69^{\mathrm{k}}$ & $69.2^{\mathrm{a}}$ & $63.51^{\mathrm{a}}$ & $15.27^{\mathrm{defgh}}$ & $4.18^{\mathrm{gh}}$ & $6.11^{\mathrm{i}}$ & $70.29^{k}$ & $65.54^{\mathrm{j}}$ & $15.0^{\mathrm{a}}$ & $4.36^{\mathrm{ij}}$ \\
\hline & 96 & B. & $6.27^{\mathrm{j}}$ & $72.10^{\mathrm{a}}$ & $67.34^{\mathrm{a}}$ & $15.33^{\text {defgh }}$ & $4.40^{\mathrm{fg}}$ & $6.38^{\mathrm{h}}$ & $77.12 \mathrm{j}$ & $72.85^{i}$ & $14.8^{\mathrm{a}}$ & $4.91^{\text {gh }}$ \\
\hline & & R.S. & $6.76^{\mathrm{i}}$ & $81.76^{\mathrm{a}}$ & $75.28^{a}$ & $15.20^{\text {defgh }}$ & $4.96^{\mathrm{e}}$ & $6.95^{\mathrm{g}}$ & $80.74^{\mathrm{hij}}$ & $75.53^{\mathrm{i}}$ & $15.4^{\mathrm{a}}$ & $4.91^{\mathrm{gh}}$ \\
\hline
\end{tabular}

- Values marked with the same letter(s) within the $2^{\text {nd }}$ interaction effects are statistically similar using Revised LSD. Test at probability $=0.05$. Lowercase refer to the $1^{\text {st }}$ interaction effect. $\mathrm{I}=$ irrigation $\left(\% \mathrm{ET} \mathrm{C}_{\mathrm{C}}\right) \cdot \mathrm{K}=\operatorname{potassium}\left(\mathrm{K}_{2} \mathrm{O}\right.$ fed $\left.^{-1}\right)$ 'M $=$ mulch type $` \mathrm{~B}=$ bare soil $`$ R.S. $=$ rice straw mulch

\section{Discussion}

The obtained results showed that, irrigation 80 and/or $100 \% \mathrm{ET}_{\mathrm{C}}$ enhanced the effect of morphological, total bulbs yield and its components over irrigation $60 \% \mathrm{ET}_{\mathrm{C}}$. Such favourable effect of irrigation at the intermediate and/or higher treatments than lower irrigation treatment might be due to better availability of moisture in root zone during entire garlic growth period and it is well known that water plays a crucial in nutrients uptake and transport and photosynthesis which favoured the growth and then reflected bulbs yield and its components [3]. Similar findings were obtained by [15] and [16] who showed that, drought is one of the major stress that adversely influences on growth and yield of crops. [17], who reported that, irrigation 80 or $100 \% \mathrm{ET}_{\mathrm{C}}$ provided, significantly, higher total and marketable bulbs yield, bulb weight, clove weight and cloves number bulb ${ }^{-1}$ than $60 \% \mathrm{ET}_{\mathrm{C}}$ of garlic.

The desirable effect of potassium rates up to $96 \mathrm{~kg} \mathrm{~K}_{2} \mathrm{O}$ fed ${ }^{-1}$ on improving growth, total bulbs yield and its components was imputed to the fact that, potassium element is producing the stocky and 
healthy appearance with thick leaves and larger bulbs of garlic plants. Also, potassium is a beneficial in soil nutrient solution and improvement absorption efficiency which reflected on stimulate on plant growth. Moreover, potassium has an essential role in translocation of synthesized carbohydrates from plant leaves towards bulbs. Typical findings on growth, total bulbs yield and its components were reported by [8] and [5] who documented that, increasing application potassium to growing garlic plants from 0 to 24 and further to 48,72 and $96 \mathrm{~kg} \mathrm{~K}_{2} \mathrm{O}$ fed ${ }^{-1}$ resulted in, significant, gradual increases on plant height, leaves number plant ${ }^{-1}$, leaf area plant $^{-1}$, dry weight plant ${ }^{-1}$, total bulbs yield, bulb weight and clove weight whereas, cloves number bulb ${ }^{-1}$ was not affected.

The promoting of rice straw mulch over bare soil on all growth, total bulbs yield and its components can be explained on basis that, using rice straw mulch depressed infiltration rate caused efficient in reduction of nutrients leaching, regulated soil temperature, much conserved soil moisture, stimulated soil flora activity, reduced weed growth, adding organic matter to soil and adequating micro atmosphere surrounding plants which reflected on growth and productivity [18] and [19]. Similar information were published on growth of garlic plants by [20] and [10] who showed that, rice straw mulch produced the highest plant height $(70.68 \mathrm{~cm})$, leaves number plant ${ }^{-1}(11.74)$, fresh leaves weight plant $^{-1}(7.51 \mathrm{~g})$ and dry weight leaves plant ${ }^{-1}(2.91 \mathrm{~g})$. Our results on bulbs yield and its components of garlic were in line with those of [10], [21] and [22] who indicated that, plants of garlic grown under rice straw mulch attained total bulbs yield $\left(5.48 \mathrm{t} \mathrm{ha}^{-1}\right)$ which was $31.41 \%$ higher than the bare soil $\left(4.17 \mathrm{t} \mathrm{ha}^{-1}\right)$.

The synergetic effect of interaction between irrigation $80 \mathrm{and} /$ or $100 \% \mathrm{ET}_{\mathrm{C}} \times$ potassium rate at $72 \mathrm{and} /$ or $96 \mathrm{~kg} \mathrm{~K}_{2} \mathrm{O}$ fed $^{-1}$ over irrigation percent at $60 \% \mathrm{ET}_{\mathrm{C}} \times$ potassium rate at $24 \mathrm{~kg} \mathrm{~K}_{2} \mathrm{O}$ fed ${ }^{1}$ on plant height, leaves number, area and dry weight plant ${ }^{-1}$, total bulbs yield, bulb weight, cloves weight and number and clove weight might be attributed to increase, as an average of both seasons, by $43.1,29.7,196.8,253.3,83.8,276.9,268.6,37.6$ and $173.4 \%$, respectively.

The favorable effect of interaction between irrigation 80 and/or $100 \% \mathrm{ET}_{\mathrm{C}} \times$ rice straw mulch over irrigation $60 \% \mathrm{ET}_{\mathrm{C}} \times$ bare soil on leaves area plant ${ }^{-1}$, in both seasons, plant height and dry weight plant ${ }^{-1}$ was attributed to increase by $83.20,22.10$ and $143.21 \%$, orderly. Again, interaction between irrigation $100 \% \mathrm{ET}_{\mathrm{C}} \times$ rice straw mulch over irrigation $60 \% \mathrm{ET}_{\mathrm{C}} \times$ bare soil on bulb weight and cloves weight bulb ${ }^{-1}$, in both seasons, cloves number bulb ${ }^{-1}$, in $1^{\text {st }}$ season was imputed to increase by $89.19,86.18$ and $16.99 \%$, respectively. However, irrigation $80 \% \mathrm{ET}_{\mathrm{C}} \times$ rice straw mulch over irrigation $60 \% \mathrm{ET}_{\mathrm{C}} \times$ bare soil on clove weight due to increase by $63.71 \%$.

The desirable effect of interaction between potassium $96 \mathrm{~kg} \mathrm{~K}_{2} \mathrm{O}$ fed $^{-1} \times$ rice straw mulch over potassium $24 \mathrm{~kg} \mathrm{~K}_{2} \mathrm{O}$ fed $^{-1} \times$ bare soil on dry weight plant ${ }^{-1}$, total bulbs yield, bulb weight, in both seasons, cloves weight bulb ${ }^{-1}$ and clove weight, in $1^{\text {st }}$ season possibly attributed to increase by $134.88,28.22,124.89,134.38$ and $97.76 \%$, respectively. However, the interaction between potassium $48 \mathrm{~kg} \mathrm{~K}_{2} \mathrm{O}$ fed- $1 \times$ rice straw mulch over potassium $24 \mathrm{~kg} \mathrm{~K}_{2} \mathrm{O}$ fed $^{-1} \times$ bare soil on cloves number bulb $^{-1}$, in $1^{\text {st }}$ season, properly to increase by $22.09 \%$.

\section{Conclusion}

Results of the current study clarified that the morphological characters and bulbs yield and its components of garlic can be improved by increasing the irrigation percent from 60 to 80 and $100 \%$ $\mathrm{ET}_{\mathrm{C}}$, fertilizing growing garlic plants by $96 \mathrm{~kg} \mathrm{~K}_{2} \mathrm{O} \mathrm{fed}{ }^{-1}$ and cover soil with rice straw. The results, also, indicated that the $1^{\text {st }}$ order interaction between irrigation percent at 100 and/or $80 \% \mathrm{ET}_{\mathrm{C}} \times$ potassium rate at 96 and/or $72 \mathrm{~kg} \mathrm{~K}_{2} \mathrm{O} \mathrm{fed}{ }^{-1}$, irrigation percent at 80 and/or $100 \% \mathrm{ET}_{\mathrm{C}} \times$ rice straw, potassium rate at $96 \mathrm{~kg} \mathrm{~K}_{2} \mathrm{O}$ fed $^{-1} \times$ rice straw and the $2^{\text {nd }}$ interaction of irrigation percent at $100 \%$ $\mathrm{ET}_{\mathrm{C}} \times$ potassium rate at $96 \mathrm{~kg} \mathrm{~K}_{2} \mathrm{O}$ fed $^{-1} \times$ rice straw mulch generally, had pronounced effects on morphological characters and bulbs yield and its components. 


\section{References}

[1] Ahmed, M. E. M., A. A. El-Aidy, A. A. Radwan and T. Sh. Abd El-Bary. 2010. Response of garlic plants to humic acid and different application methods of potassium fertilizer. Minufiya $\mathrm{J}$. Agric. Res. 35 (6): 2159 - 2175.

[2] Karam, F., R. Saliba, S. Skaf, J. Breidy, Y. Rouphael and J. Balendonck. 2011. Yield and water use of eggplants (Solanum melongena L.) under full and deficit irrigation regimes. Agric. Water Manag. 98(8): 1307 - 1316.

[3] Abd El-Latif and A. A. Abdelshafy. 2017. Response of garlic productivity to surface and drip systems and irrigation amounts. Middle East J. Agric. Res. 6 (4): 981 - 995.

[4] Mebrahtu, Y. 2017. Response of onion to deficient irrigation under different furrow irrigation water management techniques in Raya valley, Northern Ethiopia. MSc. Thesis, Univ. Hawassa, Ethiopia.

[5] El-Sayed, H. E. A. and A. H. A. El-Morsy. 2012. Response of productivity and storability of garlic (Allium sativum L.) to some potassium levels and foliar spray with mepiquat chloride (PIX). Inter. Res. J. Agric. Sci. Soil Sci. 2(7): 298 - 305.

[6] Arisha, H. M. E., S. Kh. A. Ibraheim and N. M. El-Sarkassy. 2017. Response of garlic (Allium sativum L.) yield, volatile oil and nitrate content to foliar and soil application of potassium fertilizer under sandy soil conditions. Middle East J. Appl. Sci. 7 (1): 44 - 56.

[7] El-Mansi, A. A, M. A. I. Khalil, A. A. Gad and M. H. El-Sawah. 1985. Effect of potassium fertilizer on the productivity and storage ability of garlic. Zagazig J. Agric. Res. 12 (2): 51 - 63.

[8] Shafeek, M. R., A. H. Ali, A. R. Mahmoud and M. M. Hafez. 2016. The influence of foliar and soil fertilization of potassium on growth, yield and quality of garlic plants (Allium sativum L.). Inter. J. Pharm. Tech. Res. 9(9): 390 - 397.

[9] Islam, K. S., M. H. A. Miah and S. U. Ahmed. 2010. Effect of mulch and different levels of N and $\mathrm{K}$ on the growth and yield of onion. Progress. Agric. 21 (1/2): $39-46$.

[10] Karim, M. R., M. F. Mondal and M. H. A. Rashid. 2011. Effects of NPKS and mulching on growth and yield of garlic. J. Bangladesh Soc. Agric. Sci. Technol. 8 (1/2): 119 -124.

[11] Wilde, S. A., R. B. Corey, J. G. Lyer and G. K. Voigt. 1985. Soil and plant analysis for tree culture. Oxford and IBM Publishers, New Delhi, India, 3rd ed : 93 - 106.

[12] Israelson, O. W. and V. E. Hansen. 1962. Irrigation principles and practices. Soil Sci. 95, 218.

[13] Semida, W. M. T. A. A. El-Mageed, S. E. Mohamed, and N. A. El-Sawah. 2017. Combined effect of deficit irrigation and foliar-applied salicylic acid on physiological responses, yield, and water-use efficiency of onion plants in saline calcareous soil. Arch. Agron. Soil Sci. 63 (9): 12271239.

[14] Al-Rawi, K. M. and A. M. Kalf-Allah. 1980. Design and analysis of agriculture experiments. Text book. El-Mousl Univ. Press. Ninawa, Iraq. 487.p.

[15] Boutraa, T. 2010. Improvement of water use efficiency in irrigated agriculture: A Review. J. Argon. 9 (1): $1-8$.

[16] Ghodke, P. H., P. S. Andhale, U. M. Gijare, A. Thangasamy, Y. P. Khade, V. Mahajan and M. Singh. 2018. Physiological and biochemical responses in onion crop to drought stress. Inter. J. Curr. Microbial. Appl. Sci. 7 (1): 2054 - 2062.

[17] Mandefro, C. and Q. Shoeb. 2015. Effect of deficit irrigation on yield and water productivity of garlic ( Allium Sativum L .) under drip Irrigation and mulching at Wolaita Soddo. inter. J. Life Sci. 4 (4): $232-239$. 
[18] Ghosh, P. K., D. D. bandyopadhyyay and M. Mohanty. 2006. Evaluation of straw and polythene mulch for enhancing productivity of irrigated summer groundnut. Field Crop Res. 99:76 - 86.

[19] Bhardwaj, R. L. and K. V. Kendra. 2013. Effect of mulching on crop production under rained condition - a review. Agric. Reviews, 34 (3): 188 - 197.

[20] Islam, M. J., A. K. M. M. Hossain, F. Khanam, U. K. Majumder, M. M. Rahman and M. S. Rahman. 2007. Effect of mulching and fertilization on growth and yield of garlic at Dinajpur in Bangladesh. Asian J. Plant Sic. 6(1): 98 - 101.

[21] Abouziena, H. F. and S. M. Radwan. 2015. Effects of sawdust, rice straw, bur-clover weed and cogon grass on weed control and development of onion. Inter. J. Chem. Tech. Res. 7(1): 337 - 345.

[22] Abd El-Mageed, T. A. W. M. Semida, and M. H. Abd El-Wahed. 2016. Effect of mulching on plant water status, soil salinity and yield of squash under summer-fall deficit irrigation in salt affected soil. Agric. Water Manag. (173): 1-12. 